\title{
LETTERS
}

\section{A molecular comparison of microbial communities in bronchiectasis and cystic fibrosis}

\section{To the Editor:}

Chronic bacterial infections play an important role in disease progression in patients with bronchiectasis (BX) and cystic fibrosis (CF). Although only a few per cent of all bacteria can be cultured routinely in the laboratory [1], most bacteria can be identified through sequencing the variable regions of their $16 \mathrm{~S}$ rRNA gene [2]. DNA studies in patients with CF revealed a more complex microbiota than was identified by standard culture $[3,4]$, leading us to test by sequencing whether BX may have a similarly complex polymicrobial state.

Approval was gained from the Brompton, Harefield and NHLI Research Ethics Committee and all subjects provided written consent. We collected sputa from 11 patients with BX (mean \pm SD age years $59.6 \pm 11.6$ ) and 10 with $C F$ (mean \pm SD age years $30.8 \pm 5.3$ ). An aliquot of sputum was cultured following CF Trust microbiology guidelines [5]. Following lysis and DNA extraction we amplified a hyper-variable region of the $16 \mathrm{~S}$ rRNA gene, and cloned, sequenced and analysed the products as described [6]. 96 clones were analysed for each patient. Sequences are stored as GenBank accession numbers JN212577-JN214344.

At the time of study, five of $11 \mathrm{BX}$ patients were being treated with i.v. antibiotics for an acute exacerbation. Four patients were receiving treatment for chronic fungal pulmonary disease. Other antibiotics (oral or nebulised) were being administered long term, either as long-term anti-infective agents (such as nebulised colomycin or tobramycin) or as long-term antiinflammatory immune modulators (azithromycin). Nine of the $10 \mathrm{CF}$ patients were being treated for an acute exacerbation (eight with i.v. antibiotics and one with oral antibiotics).

A total of 1768 high quality 16S rRNA gene sequences were obtained from the sputum samples of all patients. No amplification of bacterial DNA was obtained for one sample, due to the presence of an unidentified inhibitor of PCR.

A total of 48 different operational taxonomic units (OTUs) (indicating distinct bacterial sequences) was observed in the combined BX and CF samples, consisting of 42 BX group OTUs and 18 CF OTUs. 12 (25\%) OTUs were common to both groups (fig. 1). The 48 OTUs were assigned to a genus by a $>98 \%$ identity match after a BLAST (Basic Local Alignment Search Tool) search of known bacterial 16S rRNA gene sequences using RDP Release 10, update 15 [7].

Pseudomonas aeruginosa was the most common organism detected by sequencing, being present in $81 \%$ of BX and $55 \%$ of CF patients. When $P$. aeruginosa was detected in a sample it was invariably the predominant OTU present (fig. 1). Strikingly, $P$. aeruginosa was detected by sequencing in five patients with negative cultures. We found more than one Pseudomonas spp.
OTU present in two patients, supporting the observation that it is common for $\mathrm{CF}$ and $\mathrm{BX}$ patients to be infected with multiple strains of $P$. aeruginosa [8]. The $16 \mathrm{~S}$ rRNA gene cloning results did not discern between $P$. aeruginosa and its mucoid form.

In the absence of $P$. aeruginosa other organisms were predominant in the sputum samples, such as: Proteus mirabilis for patient BX4; Achromobacter sp. in patients BX7, BX10, BX15 and CF18; an unclassified Proteobacteria which grouped closely to Haemophilus influenzae and Proteus mirabilis in patient BX16; an unclassified Firmicute which grouped closely to Granulicetalla sp. and Streptococcus sp. in patient BX17; Staphylococcus aureus in patient CF2; Ralstonia sp. in patient CF21; and Stenotrophomonas maltophilia in patients CF22 and CF23. All of these organisms may be pathogens contributing to the disease process.

In only $20 \%$ of patients was there a complete agreement between bacteria detected by standard microbial culture and 16S rRNA gene sequencing. There was a partial match between $16 \mathrm{~S}$ rRNA gene sequence identity and culture results in 35\% of samples and no match in $45 \%$ of samples. This result is consistent with previous reports that $30 \%$ of purulent sputum samples from BX patients fail to culture with standard microbial techniques [9], and confirms that molecular methods can detect the significant presence of bacteria in culture negative patients [10]. Positive cultures were not accompanied by specific sequences in four patients, reflecting the limitations of small numbers of sequenced clones to identify low abundance organisms.

Amongst other common organisms, Streptococcus spp. were detected in $63 \%$ of BX patient samples and $30 \%$ of CF patient samples. Prevotella spp. were more common in BX $(73 \%)$ compared to CF (22\%). Haemophilus spp. were also present in $45 \%$ of BX and $10 \%$ of CF patients. In six patients more than one potentially pathogenic bacterium species was detected in $>30 \%$ of the sample (fig. 1).

We did not estimate bacterial load, but all patients were producing purulent sputum at the time of the study. Inspection of the OTU distribution suggested a greater species diversity in BX patients compared to CF (fig. 1), which was confirmed by Chao estimates of the mean numbers of OTUs per sputum (8.2 \pm 5.2 in $\mathrm{BX}$ patients and $2.7 \pm 2$ in patients with CF; $\mathrm{p}<0.0001$, LIBSHUFF estimate $=0.004$ ). The microbiome from both sets of specimens was markedly different from that observed in the airways of control samples from adults and children [6]. The small sample and the use of multiple antibiotics made it difficult to dissect out whether the observed levels of diversity were related to acute and chronic antibiotic use, patient age or exacerbation status.

We have studied patients at a single time in the course of a prolonged illness. The technology we have used to obtain 


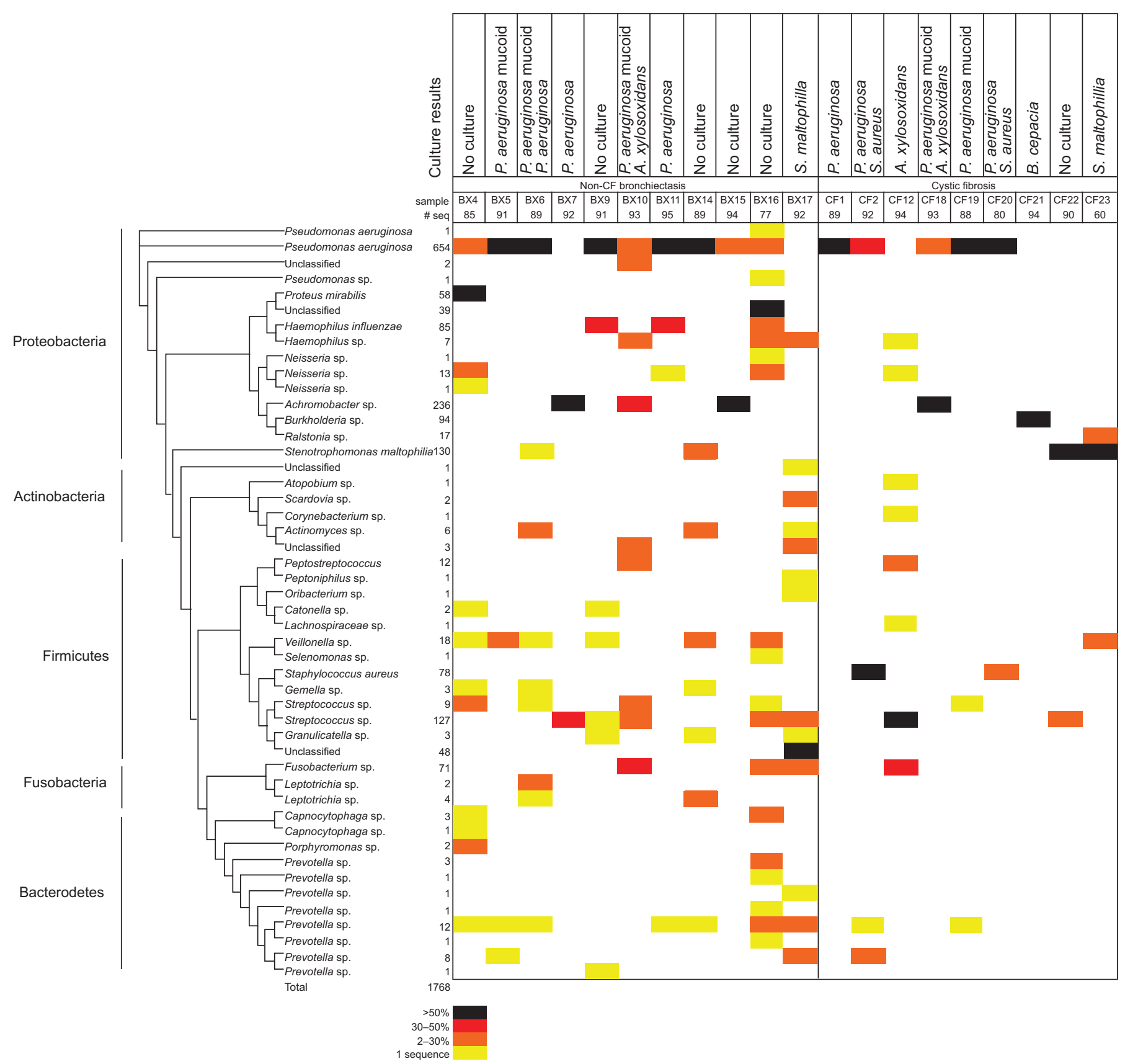

FIGURE 1. Heat map displaying the relative abundance of distinct bacterial species (defined as operational taxonomic units (OTUs)) detected by sequencing the $16 \mathrm{~S}$ rRNA gene in sputum samples from each patient. The samples are divided into two panels showing results for non-cystic fibrosis bronchiectasis (BX) and cystic fibrosis (CF). The darkness of the bars indicates the relative abundance of each species in sputum samples from each patient. A phylogenetic tree showing the relationship between OTUs is aligned with the heat map to the left and culture results observed for each sputum sample are displayed at the top of the figure.

sequences (cloning and sequencing) is cumbersome and provides small numbers, and is already being replaced by rapid and potentially high-throughput ultra-deep sequencing of amplicons of the 16S rRNA gene. Our findings are nevertheless consistent with a similar study of resected lungs from patients with these illnesses [11]. Our findings show the limitations of sputum culture in identifying all bacteria that are causal in disease progression. Our results provide hope that the integration of molecular methods into the practice of clinical microbiology will result in an improved level of diagnostic accuracy.
Rachael M. Duff*, Nicholas J. Simmonds", Jane C. Davies ${ }^{\#, q}$,

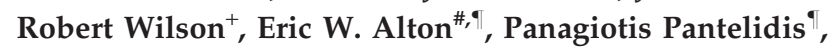

Michael J. Cox*, William O.C.M. Cookson*, Diana Bilton ${ }^{\#, \S, ~}$ Miriam F. Moffatt*,

*Molecular Genetics and Genomics Section, National Heart and Lung Institute, Imperial College, London, "Dept of Cystic Fibrosis, Royal Brompton and Harefield NHS Foundation Trust, London, 'Dept of Gene Therapy, National Heart and Lung Institute, Imperial College, London, and ${ }^{+}$Host Defence Unit, Royal 
Brompton and Harefield NHS Foundation Trust, London, UK. ${ }^{\S}$ These two authors contributed equally to the study.

Correspondence: M.F. Moffatt, Molecular Genetics and Genomics Section, National Heart and Lung Institute, Dovehouse Street, London SW3 6LY, UK. E-mail: m.moffatt @imperial.ac.uk

Support Statement: The study was supported by the NIHR Respiratory Disease Biomedical Research Unit at the Royal Brompton and Harefield NHS Foundation Trust and Imperial College London, by a Research Excellence Award from Imperial College London, and by the Wellcome Trust (Grant number 077959/Z/05/Z).

Statement of Interest: None declared.

Acknowledgements: We are grateful to Michael Loebinger (Host Defence Unit, Royal Brompton and Harefield NHS Foundation Trust, London and Molecular Genetics and Genomics Section, National Heart and Lung Institute, Imperial College, London, UK) for his thoughtful comments on the paper.

\section{REFERENCES}

1 Staley JT, Konopka A. Measurement of in situ activities of nonphotosynthetic microorganisms in aquatic and terrestrial habitats. Annu Rev Microbiol 1985; 39: 321-346.
2 Turnbaugh PJ, Ley RE, Hamady M, et al. The human microbiome project. Nature 2007; 449: 804-810.

3 Rogers GB, Carroll MP, Serisier DJ, et al. Bacterial activity in cystic fibrosis lung infections. Respir Res 2005; 6: 49.

4 Cox MJ, Allgaier M, Taylor B, et al. Airway microbiota and pathogen abundance in age-stratified cystic fibrosis patients. PLoS One 2010; 5: e11044.

5 Saiman L, Siegel J. Infection control recommendations for patients with cystic fibrosis: microbiology, important pathogens, and infection control practices to prevent patient-to-patient transmission. Infect Control Hosp Epidemiol 2003; 24: S6-S52.

6 Hilty M, Burke C, Pedro H, et al. Disordered microbial communities in asthmatic airways. PLoS One 2010; 5: e8578.

7 Cole JR, Wang Q, Cardenas E, et al. The Ribosomal Database Project: improved alignments and new tools for rRNA analysis. Nucleic Acids Res 2009; 37: D141-D145.

8 Waine DJ, Honeybourne D, Smith EG, et al. Cross-sectional and longitudinal multilocus sequence typing of Pseudomonas aeruginosa in cystic fibrosis sputum samples. J Clin Microbiol 2009; 47: 3444-3448.

9 King PT. The pathophysiology of bronchiectasis. Int J Chron Obstruct Pulmon Dis 2009; 4: 411-419.

10 Rogers GB, Daniels TW, Tuck A, et al. Studying bacteria in respiratory specimens by using conventional and molecular microbiological approaches. BMC Pulm Med 2009; 9: 14.

11 Maughan $\mathrm{H}$, Cunningham KS, Wang PW, et al. Pulmonary bacterial communities in surgically resected noncystic fibrosis bronchiectasis lungs are similar to those in cystic fibrosis. Pulm Med 2012; 2012: 746358.

\section{Exacerbation of respiratory symptoms in COPD patients may not be exacerbations of COPD}

\section{To the Editor:}

Exacerbations of chronic obstructive pulmonary disease (COPD) are defined as acute events characterised by a worsening of the patient's respiratory symptoms, particularly dyspnoea, beyond day-to-day variation, leading to a change in medical treatment and/or hospitalisation [1, 2]. Exacerbations of COPD are a leading cause of hospitalisation and healthcare expenditures, particularly in frail, elderly patients. They alter the health-related quality of life and the natural course of disease, increasing the risk of mortality, both during and after the acute event $[1,2]$. Patients with COPD frequently have chronic comorbidities [1]. Several of these comorbidities may produce acute events, contributing to the increased morbidity and mortality in COPD exacerbations: acute myocardial infarction, congestive heart failure, cerebrovascular disease, cardiac arrhythmias and pulmonary circulation disorders [1].

By definition, acute exacerbations of COPD are considered respiratory diseases, with specific reference to the respiratory symptoms and to the organs involved (airways and lung). Indeed, respiratory viral or bacterial infections and air pollution are assumed to be the main causes of COPD exacerbations, but the exact contribution of infections is difficult to establish, and the aetiology of a large proportion of exacerbations remains undetermined $[1,3,4]$.

Although it is known that bronchoconstriction and hyperinflation contribute to the increase in dyspnoea in COPD patients, the chronic airway, pulmonary and systemic inflammation present in patients with stable COPD is associated with an acute transient inflammatory process when respiratory symptoms are exacerbated by infections and/or pollutants. Neutrophils and/or eosinophils increase in the airways and lung, together with inflammatory mediators and protein leakage (fig. 1). This acute "respiratory" inflammation is associated with systemic inflammation, as shown by the increase in circulating inflammatory cells and pro-inflammatory cytokines. These systemic effects represent an important pathogenic link between COPD and comorbidities, particularly cardiovascular diseases [3, 4]. Indeed, epidemics of influenza are associated with an increased risk of death, particularly in elderly patients with chronic diseases, and the cause of death may be both respiratory and cardiovascular, particularly in the case of myocardial infarctions and stroke [5]. 